\title{
Sometimes a stick might just be a stick
}

\author{
Christopher B. Sturdy ${ }^{1,2}$. Jenna V. Congdon ${ }^{1}$
}

Published online: 3 April 2019

(C) The Psychonomic Society, Inc. 2019

\begin{abstract}
Suzuki (Proceedings of the Natural Academy of Sciences, 115, 1541-1545, 2018) conducted elegant field experiments examining referential communication in Japanese tits. Bond (Learning \& Behavior, in press, 2019) explains some key considerations and future experimentation that should be conducted to solidify these conclusions. An important takeaway from both Suzuki and Bond is that scientists can, and should, both be excited for new, interesting scientific discoveries, and also view such findings with a critical, but collegial, eye for more parsimonious explanations and the manipulations required to test such explanations.
\end{abstract}

Keywords Parids $\cdot$ Alarm calls $\cdot$ Referential communication

\section{Introduction}

When Suzuki (2018) published the latest in his line of enviable studies on wild Japanese tits (Parus minor), we read it with great anticipation, and not a little dose of envy. Here, and in his research program, Suzuki has identified, and seemingly mastered, interesting and important field work with a member of our current study genus: the Parids. Admittedly we do not know as much as we should about the European and Asian cousins of the North American species that have occupied a place in our hearts for nearly 25 years of cognition and communication research (recent work summarized in Hahn et al., 2017; McMillan et al., 2017). From the most recent experiments in a line of interesting studies conducted with Asian Parids, Suzuki seemed to have convincingly demonstrated, to us, reviewers, and at least one editor of a high-profile journal, that Japanese tits use referential communication. However, after reading and re-reading a thoughtful and circumspect reconsideration of Suzuki's (2018) study, written by Alan Bond, we must admit that we may have been too hasty in our acceptance of Suzuki's conclusions, even in the face of a later thoughtful reply by Suzuki (2019).

Christopher B. Sturdy

csturdy@ualberta.ca

1 Department of Psychology, University of Alberta, Edmonton, Alberta, Canada

2 Neuroscience and Mental Health Institute, University of Alberta, Edmonton, Alberta, Canada

\section{Japanese tit vocalizations and usage}

For the purpose of understanding the original work of Suzuki (2018), we will only briefly summarize the Japanese tits' relevant vocalizations. Chicka, or "general alarm calls" as Suzuki calls them, are produced in response to a wide array of predators, while the distinctive jar or snake-specific calls are produced in response to snakes, a main predator of Japanese tits. Third, recruitment calls are produced in non-predatory contexts such as foraging. Importantly, the two acoustically distinct predatorrelated vocalizations lead to distinct behavioral responses by tits. Chicka calls result in birds searching for a predator in the horizontal plane, while jar calls result in birds looking to the ground for a snake. There are more details and nuances, but this snapshot is all that is needed to understand the experiment and arguments.

\section{The current experiment}

Owing to the seemingly functionally referential nature (Jar calls or snake-specific alarm, chicka calls or general alarm calls), Suzuki designed two experiments to determine whether he could collect evidence for such referentiality. The experiment was such that birds hearing a call that signalled a particular type of predator should be more prepared to detect, and act upon, the signalled predator type, and should be quantifiable. The first experiment examined tits' reactions to a stick (used as a proxy for a model snake) when moved up a tree in a snake-like manner following the playback of: (1) snake-specific, (2) general alarm, or (3) recruitment calls. Following snake-specific but not general or recruitment calls, birds reacted by moving closer to the stick. Experiment 2 followed a similar procedure to Experiment 1, 
but the stick was moved along the ground in a snake-like manner or swung from a shrub in a non-snake-like movement pattern. In Experiment 2, as in Experiment 1, birds reacted by approaching the stick only when moved along the ground, and only when paired with snake-specific calls.

\section{Suzuki's conclusions}

Suzuki's conclusions to his elegantly designed experiment followed his clear results: snake-specific calls activated a mental representation of a snake and resulted in approach of the stick models when moved in a snake-like fashion. Playback of other, non-snake-specific calls did not evoke the same response. We too, concluded similarly, both upon first reading the paper, and again when reading Suzuki's commentary for Learning \& Behavior. However exciting this finding appears to be at first blush, reading Bond's careful and fair commentary has caused us to rethink possible alternative explanations of Suzuki's results.

\section{Bond's conclusions}

In his commentary, Bond (2019) is more circumspect, and while not dismissing out of hand the notion of representations, referents, or search images, reminds us that there may be simpler explanations at hand. For instance, the fact that the two different call types lead tits to look at the sky or the ground could form the basis for their response by directing attention towards or away from the stick. It should be noted here that there seems to be a difference of interpretation. Suzuki (2014) reported that tits move their heads horizontally when scanning for a threat. Bond's (2019) interpretation is first that birds "move their heads horizontally, scanning the sky and nearby trees" and later states that "If the innate response to chicka calls is to stare at the sky, while that to jar calls is to scan the ground..." This difference of interpretation will need to be resolved at a later date.

After identifying these key points, Bond describes two critical control experimental manipulations that would need to be conducted before the referential/representational/search image debate is settled. The first involves testing the snake model to ensure that the model itself leads to anti-snake behavior in the absence of anti-snake call playback. The second involves manipulating various parameters of the model to determine how they factor into the tits' response. Collegially, Bond then outlines the very experimental conditions that Suzuki (or anyone else) would need to run in order to address these concerns.

\section{So now what should the rest of us think?}

The title of this subsection might sound like we are about to write what we feel others should think about the initial experiment by
Suzuki and the subsequent critique by Bond. In fact, we write this section as much for ourselves as we do for anyone else.

We should be skeptical of experimental results, especially those that are highly provocative or that confirm our biases or predilections. This does not mean that we should be overly critical or hostile, but rather that we should attempt to learn as much as possible about the experiments, the results and conclusions, and then we ought to apply Occam's Razor in order to determine whether a simpler, more parsimonious explanation will serve as well as a more complicated explanation.

\section{Final thoughts}

Suzuki (2018) conducted an elegant and enviable pair of field experiments that seem to suggest that Japanese tits have mental representations of predators. A careful and thoughtful critique by Bond (2019) calls for additional experimentation before passing any final judgments, for or against, Suzuki's results. Both Suzuki (2018, 2019) and Bond (2019) make excellent points, and we as a scientific community would do well to withhold final judgment until further experiments are conducted. We would be excited to learn the results of these experiments.

\section{References}

Bond, A. (2019). Searching images and the meaning of alarm calls. Learning \& Behavior.

Hahn, A. H., Congdon, J. V., Campbell, K. A., Scully, E. N., McMillan, N., \& Sturdy, C. B. (2017). Mechanisms of communication and cognition in chickadees: Explaining nature in the lab and field. In Advances in the Study of Behavior (Vol. 49, pp. 147-197). Academic Press, Cambridge.

McMillan, N., Avey, M. T., Bloomfield, L. L., Guillette, L. M., Hahn, A. H., Hoeschele, M., \& Sturdy, C. B. (2017). Avian vocal perception: Bioacoustics and perceptual mechanisms. In C. ten Cate \& S. D. Healy (Eds) Avian Cognition. Cambridge: Cambridge University Press.

Suzuki, T. N. (2014). Communication about predator type by a bird using discrete, graded and combinatorial variation in alarm calls. Animal Behaviour, 87, 59-65.

Suzuki, T. N. (2018). Alarm calls evoke a visual search image of a predator in birds. Proceedings of the Natural Academy of Sciences, 115, 1541-1545.

Suzuki, T. N. (2019). Imagery in wild birds: Retrieval of visual information from referential alarm calls. Learning \& Behavior.

Publisher's note Springer Nature remains neutral with regard to jurisdictional claims in published maps and institutional affiliations. 